\title{
Word Level Predicate Abstraction and Refinement for Verifying RTL Verilog
}

\author{
Himanshu Jain \\ CMU SCS, Pittsburgh, PA 15213 \\ Natasha Sharygina \\ CMU SEI, Pittsburgh, PA 15213
}

\author{
Daniel Kroening \\ ETH Zürich, Switzerland \\ Edmund Clarke \\ CMU SCS, Pittsburgh, PA 15213
}

\begin{abstract}
Model checking techniques applied to large industrial circuits suffer from the state space explosion problem. A major technique to address this problem is abstraction. The most commonly used abstraction technique for hardware verification is localization reduction, which removes latches that are not relevant to the property. However, localization reduction fails to reduce the size of the model if the property actually depends on most of the latches. This paper proposes to use predicate abstraction for verifying RTL Verilog, a technique successfully used for software verification. The main challenge when using predicate abstraction is the discovery of suitable predicates. We propose to use weakest pre-conditions of Verilog statements in order to obtain new predicates during abstraction refinement. This technique has not been applied to circuits before. On benchmarks taken from an industrial microprocessor, we successfully verified safety properties with more than 32,000 latches in the cone of influence. We compare the performance of our technique with a modern model checker that implements localization reduction.
\end{abstract}

Categories and Subject Descriptors: B.5.2 [Hardware]: RegisterTransfer-Level Implementation-Design Aids; J.6 [Computer Aided Engineering]: [Computer-Aided Design]

General Terms: Verification

Keywords: Predicate Abstraction, Verilog, SAT

\section{INTRODUCTION}

Model checking [8] is one of the most commonly used formal verification techniques in a commercial setting. However, model checking suffers from the state space explosion problem. One principal method in state space reduction is abstraction. Abstraction techniques reduce the state space by mapping the set of states of

\footnotetext{
* This research was sponsored by the Gigascale Systems Research Center (GSRC), the National Science Foundation (NSF) under grant no. CCR9803774, the Office of Naval Research (ONR), the Naval Research Laboratory (NRL) under contract no. N00014-01-1-0796, and by the Defense Advanced Research Projects Agency, and the Army Research Office (ARO) under contract no. DAAD19-01-1-0485.
}

Permission to make digital or hard copies of all or part of this work for personal or classroom use is granted without fee provided that copies are not made or distributed for profit or commercial advantage and that copies bear this notice and the full citation on the first page. To copy otherwise, to republish, to post on servers or to redistribute to lists, requires prior specific permission and/or a fee.

DAC 2005, June 13-17, 2005, Anaheim, California, USA.

Copyright 2005 ACM 1-59593-058-2/05/0006 ...\$5.00. the actual, concrete system to an abstract, and smaller, set of states in a way that preserves the relevant behaviors of the system.

In the hardware domain, the most commonly used abstraction technique is localization reduction $[16,21,5]$. The abstract model is created from the given circuit by removing a large number latches together with the logic required to compute their next state. The latches that are removed are called the invisible latches. The latches remaining in the abstract model are called visible latches. The initial abstract model is created by making the latches present in the property as visible, and the rest as invisible.

Localization reduction is a conservative over-approximation of the original circuit for reachability properties. This implies that if the abstraction satisfies the property, the property also holds on the original, concrete circuit. The drawback of the conservative abstraction is that when model checking of the abstraction fails, it may produce a counterexample that does not correspond to any concrete counterexample. This is usually called a spurious counterexample.

In order to check if an abstract counterexample is spurious, the abstract counterexample is simulated on the concrete machine. This is called the simulation step. Like in Bounded Model Checking (BMC), the concrete transition relation for the design and the given property are jointly unwound to obtain a Boolean formula. The number of unwinding steps is given by the length of the abstract counterexample. As in BMC, the Boolean formula is then checked for satisfiability using a SAT procedure [21]. If the instance is satisfiable, the counterexample is real and the algorithm terminates. If the instance is unsatisfiable, the abstract counterexample is spurious, and abstraction refinement has to be performed.

The basic idea of the abstraction refinement techniques is to create a new abstract model which contains more detail in order to prevent the spurious counterexample. This process is iterated until the property is either proved or disproved. It is known as the Counterexample Guided Abstraction Refinement framework, or CEGAR for short [16, 3, 6, 13, 21].

In the software domain, the most successful abstraction technique for large systems is predicate abstraction [14]. It abstracts data by only keeping track of certain predicates on the data. Each predicate is represented by a Boolean variable in the abstract program, while the original data variables are eliminated. When applying predicate abstraction to circuits, two problems arise:

- Most model-checkers used in the hardware industry work on a very low level design, usually a net-list. However, predicate abstraction is only effective if the predicates can cover the relationship between multiple latches. This typically requires a word level model given in register transfer language (RTL), e.g., in Verilog. The RTL level languages are similar to languages used in the software domain, such as ANSI-C. 


\section{Report Documentation Page}

Public reporting burden for the collection of information is estimated to average 1 hour per response, including the time for reviewing instructions, searching existing data sources, gathering and maintaining the data needed, and completing and reviewing the collection of information. Send comments regarding this burden estimate or any other aspect of this collection of information, including suggestions for reducing this burden, to Washington Headquarters Services, Directorate for Information Operations and Reports, 1215 Jefferson Davis Highway, Suite 1204, Arlington VA 22202-4302. Respondents should be aware that notwithstanding any other provision of law, no person shall be subject to a penalty for failing to comply with a collection of information if it does not display a currently valid OMB control number.

\begin{tabular}{|c|c|c|}
\hline $\begin{array}{l}\text { 1. REPORT DATE } \\
\mathbf{2 0 0 5}\end{array}$ & 2. REPORT TYPE & $\begin{array}{l}\text { 3. DATES COVERED } \\
\mathbf{0 0 - 0 0 - 2 0 0 5} \text { to 00-00-2005 }\end{array}$ \\
\hline \multirow{3}{*}{\multicolumn{2}{|c|}{$\begin{array}{l}\text { Word Level Predicate Abstraction and Refinement for Verifying RTL } \\
\text { Verilog }\end{array}$}} & 5a. CONTRACT NUMBER \\
\hline & & 5b. GRANT NUMBER \\
\hline & & 5c. PROGRAM ELEMENT NUMBER \\
\hline \multirow{3}{*}{\multicolumn{2}{|c|}{ 6. AUTHOR(S) }} & 5d. PROJECT NUMBER \\
\hline & & 5e. TASK NUMBER \\
\hline & & 5f. WORK UNIT NUMBER \\
\hline \multicolumn{2}{|c|}{$\begin{array}{l}\text { 7. PERFORMING ORGANIZATION NAME(S) AND ADDRESS(ES) } \\
\text { Carnegie Mellon University,Software Engineering Institute,5000 Forbes } \\
\text { Avenue,Pittsburgh,PA,15213-3890 }\end{array}$} & $\begin{array}{l}\text { 8. PERFORMING ORGANIZATION } \\
\text { REPORT NUMBER }\end{array}$ \\
\hline \multirow{2}{*}{\multicolumn{2}{|c|}{ 9. SPONSORING/MONITORING AGENCY NAME(S) AND ADDRESS(ES) }} & 10. SPONSOR/MONITOR'S ACRONYM(S) \\
\hline & & $\begin{array}{l}\text { 11. SPONSOR/MONITOR'S REPORT } \\
\text { NUMBER(S) }\end{array}$ \\
\hline
\end{tabular}

12. DISTRIBUTION/AVAILABILITY STATEMENT

Approved for public release; distribution unlimited

13. SUPPLEMENTARY NOTES

14. ABSTRACT

Model checking techniques applied to large industrial circuits suffer from the state space explosion problem. A major technique to address this problem is abstraction. The most commonly used abstraction technique for hardware verification is localization reduction, which removes latches that are not relevant to the property. However, localization reduction fails to reduce the size of the model if the property actually depends on most of the latches. This paper proposes to use predicate abstraction for verifying RTL Verilog, a technique successfully used for software verification. The main challenge when using predicate abstraction is the discovery of suitable predicates. We propose to use weakest pre-conditions of Verilog statements in order to obtain new predicates during abstraction refinement. This technique has not been applied to circuits before. On benchmarks taken from an industrial microprocessor, we successfully verified safety properties with more than 32,000 latches in the cone of influence. We compare the performance of our technique with a modern model checker that implements localization reduction.

15. SUBJECT TERMS

16. SECURITY CLASSIFICATION OF:

a. REPORT

unclassified b. ABSTRACT unclassified c. THIS PAGE unclassified
17. LIMITATION OF ABSTRACT

Same as Report (SAR)
18. NUMBER OF PAGES

6 19a. NAME OF

RESPONSIBLE PERSON 
- The second problem concerns the use of theorem provers for computing the predicate abstraction. Theorem provers model the variables using unbounded integer numbers. Overflow or bit-wise operators are not modeled. However, hardware description languages like Verilog provide an extensive set of bitwise operators. For hardware designs, the use of these bit-level constructs is ubiquitous.

Predicate abstraction tools used for software employ multiple heuristics in order to reduce the cost of calling the theorem prover while computing the abstraction. SLAM [3] applies ad-hoc heuristics that limit the number of predicates in a query, i.e., it partitions the set of predicates into smaller subsets. This speeds up the abstraction process, but the resulting abstraction contains additional spurious behavior. If the SLAM toolkit encounters a spurious counterexample, it first assumes that it is caused by a lack of predicates, and attempts to find new predicates. If no new predicates are found, SLAM concludes that the counterexample is caused by the partitioning of the predicates during the abstraction. In this case, a separate refinement algorithm (called Constrain [2]) is invoked. This step only addresses spurious behavior due to an inexact abstraction, as opposed to spurious behavior caused by insufficient predicates.

In the BLAST tool [15], the abstraction is completely demanddriven. Initially, BLAST uses a very coarse abstraction. Additional abstraction is only performed when a spurious counterexample is encountered. The abstraction is only done to the extent necessary to remove the spurious behavior. This is called lazy abstraction.

Contribution. This paper introduces new techniques for wordlevel predicate abstraction and refinement for circuits given in Verilog RTL. There are two challenges when applying predicate abstraction to circuits: 1) The computation of the abstract model is hard in presence of large number of predicates, and 2) predicate abstraction relies on a good predicate discovery algorithm.

In order to address the first problem, we partition the set of predicates into clusters of related predicates. The abstraction is computed separately with respect to the predicates in each cluster. Since each cluster contains only a small number of predicates, the computation of the abstraction becomes more efficient. We refer to this technique as predicate partitioning. As a result of the partitioning, additional spurious counterexamples are introduced which have to be removed during the refinement phase. In this context, we identify lazy abstraction [15] and eager abstraction [10] as special cases of predicate partitioning. The eager technique refers to the case when all predicates are within a single cluster, while lazy abstraction corresponds to the case in which none or very few predicates are used for computing the abstract model.

As in [10], we use SAT to compute the abstract transition relation. However, the predicate partitioning technique is also applicable with any other solver (or theorem prover).

When a spurious counterexample is encountered, we first check whether each transition in the counterexample can be simulated on the original program. This is done by creating a SAT instance for the simulation of each abstract transition. If the SAT instance for an abstract transition is unsatisfiable, then the abstract transition is spurious. In this case, we refine the abstraction by adding constraints on the abstract transition relation which eliminates the spurious transition. We make use of the proof of unsatisfiability of the SAT instance to extract a small set of predicates to eliminate the transition. The fewer predicates are found, the more spurious counterexamples can be eliminated in one step.

When all instances are satisfiable it implies that none of the abstract transitions is spurious due to the partitioning. The immediate conclusion then is that the spurious counterexample is caused by

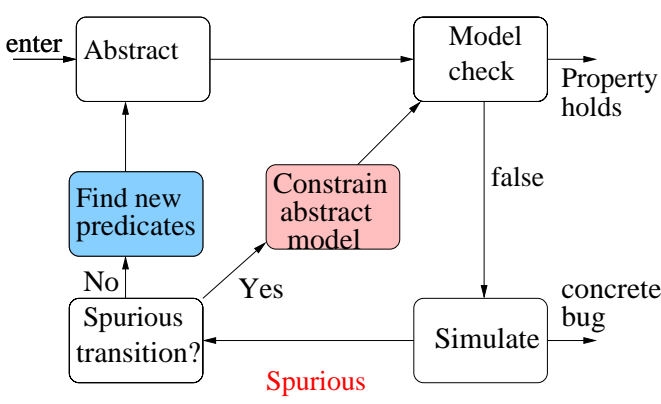

Figure 1: Abstraction-refinement loop in this paper.

insufficient predicates. In this case, we apply a novel word-level refinement technique: we compute the weakest precondition of the property with respect to the transition function given by the circuit. To the best of our knowledge, this is the first time syntactic weakest preconditions of circuits have been used for refinement in predicate abstraction. The overall flow of the various techniques described above is shown in Fig. 1.

Related work. In [11], a SAT-based technique for predicate abstraction of circuits given in Verilog is introduced. The circuit is synthesized and transformed into net-list level. A SAT solver like ZChaff [17] is used in order to perform the abstraction, which allows to support all bit-level constructs. However, if refinement becomes necessary, only bit-level predicates are introduced.

Andraus et al. [1] present a scheme for automatic abstraction of behavioral RTL Verilog to the CLU language used by the UCLID system [4]. However, the abstractions produced by their approach can be coarse as there is no direct support for bit-vectors and bitwise operators in the CLU language. Also no refinement is done when a spurious counterexample is obtained.

Outline. In section 2, we provide the notation used throughout the paper. Section 3 describes the SAT-based predicate abstraction. Techniques for partitioning predicates are given in section 4. We present techniques for word-level abstraction refinement in section 5. We report the experimental results in section 6, and conclude the paper in section 7 . The formal semantics of the subset of Verilog we handle can be found in our technical report [9].

\section{PRELIMINARIES}

Let $\mathcal{R}=\left\{r_{1}, \ldots, r_{n}\right\}$ denote the set of registers. The state of the Verilog program is given by the valuation of these registers. We consider the external inputs to be registers without a next-state function. Let $Q \subseteq \mathcal{R}$ denote the set of registers that are not external inputs, i.e., have a next-state function. We denote the next-state function of a word-level register $r_{i} \in Q$ by $f_{i}\left(r_{1}, \ldots, r_{n}\right)$, or $f_{i}(\bar{r})$ using vector notation. The transition relation $R\left(\bar{r}, \bar{r}^{\prime}\right)$ relates the current state $\bar{r} \in S$ to the next state $\bar{r}^{\prime}$ and is defined as follows:

$$
R\left(\bar{r}, \bar{r}^{\prime}\right):=\bigwedge_{r_{i} \in Q}\left(r_{i}^{\prime} \Leftrightarrow f_{i}(\bar{r})\right)
$$

Example: Consider a register $x$ of size 8 bits. In each clock cycle, if $x$ is less than five, then the value of $x$ is incremented by two, else the value of $x$ remains unchanged. Thus, the next state function of $x$ is given by $((x<5) ?(x+2): x)$, where ? denotes the choice operator. Note that we have a next state function for the whole register $x$ and not for the individual bits in $x$.

We follow the counterexample guided abstraction refinement (CEGAR) framework to prove the given property. The first step of the CEGAR loop is to obtain an abstraction of the given program. We use SAT-based predicate abstraction for this purpose. 


\section{PREDICATE ABSTRACTION}

In predicate abstraction [14], the variables of the concrete program are replaced by Boolean variables that correspond to a predicate on the variables in the concrete program. These predicates are functions that map a concrete state $\bar{r} \in S$ into a Boolean value. Let $\mathrm{B}=\left\{\pi_{1}, \ldots, \pi_{\mathrm{k}}\right\}$ be the set of predicates over the given program. When applying all predicates to a specific concrete state, one obtains a vector of Boolean values, which represents an abstract state $\bar{b}$. We denote this function by $\alpha(\bar{r})$. It maps a concrete state into an abstract state and is therefore called an abstraction function.

We perform an existential abstraction [7], i.e., the abstract model can make a transition from an abstract state $\bar{b}$ to $\bar{b}^{\prime}$ iff there is a transition from $\bar{r}$ to $\bar{r}^{\prime}$ in the concrete model and $\bar{r}$ is abstracted to $\bar{b}$ and $\bar{r}^{\prime}$ is abstracted to $\bar{b}^{\prime}$. We call the abstract machine $\hat{T}$, and we denote the transition relation of $\hat{T}$ by $\hat{R}$.

$$
\hat{R}:=\left\{\left(\bar{b}, \bar{b}^{\prime}\right) \mid \exists \bar{r}, \bar{r}^{\prime} \in S: \quad \begin{array}{rl} 
& R\left(\bar{r}, \bar{r}^{\prime}\right) \wedge \\
& \left.\alpha(\bar{r})=\bar{b} \wedge \alpha\left(\bar{r}^{\prime}\right)=\bar{b}^{\prime}\right\}
\end{array}\right.
$$

The initial state $I(\bar{r})$ is abstracted as follows:

$$
\hat{I}(\bar{b}):=\exists \bar{r} \in S:(\alpha(\bar{r})=\bar{b}) \wedge I(\bar{r})
$$

The abstraction of a safety property $P(\bar{r})$ is defined as follows: for the property to hold on an abstract state $\bar{b}$, the property must hold on all states $\bar{r}$ that are abstracted to $\bar{b}$.

$$
\hat{P}(\bar{b}):=\forall \bar{r} \in S:(\alpha(\bar{r})=\bar{b}) \Rightarrow P(\bar{r})
$$

Thus, if $\hat{P}$ holds on all reachable states of the abstract model, $P$ also holds on all reachable states of the concrete model.

SAT-based predicate abstraction. In [10], a SAT solver is used to compute the abstraction of a sequential ANSI-C program. This approach supports all ANSI-C integer operators, including the bit-vector operators. We use a similar technique for computing the abstraction of the Verilog programs. A symbolic variable $b_{i}$ is associated with each predicate $\pi_{i}$. Each concrete state $\bar{r}=\left\{r_{1}, \ldots, r_{n}\right\}$ maps to an abstract state $\bar{b}=\left\{b_{1}, \ldots, b_{k}\right\}$, where $b_{i}=\pi_{i}(\bar{r})$. If the concrete machine makes a transition from state $\bar{r}$ to state $\bar{r}^{\prime}=$ $\left\{r_{1}^{\prime}, \ldots, r_{n}^{\prime}\right\}$, then the abstract machine makes a transition from state $\bar{b}$ to $\bar{b}^{\prime}=\left\{b_{1}^{\prime}, \ldots, b_{k}^{\prime}\right\}$, where $b_{i}^{\prime}=\pi_{i}\left(\bar{r}^{\prime}\right)$.

The formula that is passed to the SAT solver directly follows from the definition of the abstract transition relation $\hat{R}$ as given in equation 1 . The set of abstract transitions $\hat{R}$ is computed by transforming equation 1 into conjunctive normal form (CNF) and passing the resulting formula to a SAT solver. The satisfying assignments obtained form the abstract transition relation $\hat{R}$.

Example: Let the transition relation $R\left(x, y, x^{\prime}, y^{\prime}\right)$ be $x^{\prime}=y \wedge y^{\prime}=$ $x$. Let the set of predicates be $\{x=1, y=1\}$. The equation for computing the $\hat{R}$ is given as follows:

$$
\begin{aligned}
& \exists x, y, x^{\prime}, y^{\prime}:\left(b_{1} \Leftrightarrow(x=1)\right) \wedge\left(b_{2} \Leftrightarrow(y=1)\right) \wedge \\
& R\left(x, y, x^{\prime}, y^{\prime}\right) \wedge\left(b_{1}^{\prime} \Leftrightarrow\left(x^{\prime}=1\right)\right) \wedge\left(b_{2}^{\prime} \Leftrightarrow\left(y^{\prime}=1\right)\right)
\end{aligned}
$$

The set of satisfying assignments to the above equation results in $\hat{R}:=\left(\left(b_{1}^{\prime} \Leftrightarrow b_{2}\right) \wedge\left(b_{2}^{\prime} \Leftrightarrow b_{1}\right)\right)$.

Note that the predicates used for abstraction can be arbitrary Boolean expressions allowed by the Verilog syntax. Thus, the predicates can involve operators for concatenation, extraction etc. For example, a $[3: 0]>7, \operatorname{ram}\left[\left\{\mathrm{addr}, 1^{\prime} \mathrm{b} 0\right\}\right]==d[9: 2]$ are allowed as predicates.

\section{PREDICATE PARTITIONING}

We call the computation of the exact existential abstraction as described in the previous section the Eager approach. In the worst case, the number of satisfying assignments is exponential in the number of predicates. In practice, computing abstractions using the eager approach can be very slow even for a small number of predicates.

The speed of the abstraction computation can be improved if we do not aim at the the most precise abstract transition relation. That is, we allow our abstraction to be an over-approximation of the abstract transition relation generated by the eager approach. The SLAM toolkit, for example, limits the number of predicates in each theorem prover query. Thus, the set of the predicates and their next-state state versions is partitioned into smaller sets of related predicates. We call these sets clusters, and denote them by $C_{1}, \ldots, C_{l}$, with $C_{j} \subseteq\left\{\pi_{1}, \ldots, \pi_{k}, \pi_{1}^{\prime}, \ldots, \pi_{k}^{\prime}\right\}$, where $\pi_{i}^{\prime}$ denotes the next state version of $\pi_{i}$.

The equation for abstracting the transition system with respect to $C_{j}$ is given as follows:

$$
\exists \bar{r}, \bar{r}^{\prime}: \bigwedge_{\pi_{i} \in C_{j}} b_{i}=\pi_{i}(\bar{r}) \wedge R\left(\bar{r}, \bar{r}^{\prime}\right) \wedge \bigwedge_{\pi_{i}^{\prime} \in C_{j}} b_{i}^{\prime}=\pi_{i}\left(\bar{r}^{\prime}\right)
$$

The satisfying assignments to the above equation correspond to the abstract transition relation $\hat{R}_{j}$, which is represented symbolically using BDDs. The number of satisfying assignments to the above equation is limited by size of cluster $C_{j}$, that is, $2^{\left|C_{j}\right|}$. Clearly, by limiting the size of $C_{j}$, we can compute the abstract transition relations much faster as compared to the eager approach.

The conjunction of $l$ abstract transition relations $\hat{R}_{1}, \ldots, \hat{R}_{l}$ results in the abstract transition relation $\hat{R}$ :

$$
\hat{R}:=\bigwedge_{i=1}^{l} \hat{R}_{i}
$$

We refer to the above technique of partitioning the set of predicates in various clusters, and using these clusters for computing the abstraction $\hat{R}$, as predicate partitioning.

Claim. If $\hat{Q}$ denotes the transition relation obtained by using the eager approach (Eqn. 1), and $\hat{R}$ denotes the transition relation obtained by predicate partitioning (Eqn. 2), then $\hat{Q} \Rightarrow \hat{R}$.

The above claim is proved by observing that for all $1 \leq j \leq l$, $\hat{Q} \Rightarrow \hat{R}_{j}$. Thus, $\hat{R}$ is an over-approximation of $\hat{Q}$, and hence, a conservative over-approximation of the original circuit.

We evaluate two different techniques for creating predicate clusters used in predicate partitioning, cone partitioning and partitioning for lazy abstraction.

Syntactic cone partitioning. This technique clusters a next state predicate with a set of current state predicates if the variables appearing in the current state predicates affect the value of the next state predicate.

Example: Let the transition relation $R\left(x, y, x^{\prime}, y^{\prime}\right)$ be $x^{\prime}=y \wedge y^{\prime}=x$. Let the set of predicates be $\left\{x=1, y=1, x^{\prime}=1, y^{\prime}=1\right\}$. The value of the predicate $y^{\prime}=1$ is affected by the value of $x$ (as $y^{\prime}$ equals $x$ ). Note that the value of $y^{\prime}=1$ is not affected by the value of $y$. Thus, we keep $x=1$ and $y^{\prime}=1$ together in a cluster $C_{1}$. Similarly, the other cluster $C_{2}:=\left\{y=1, x^{\prime}=1\right\}$ is obtained.

Syntactic partitioning for lazy abstraction. The idea of lazy abstraction [15] is to defer the abstraction until required by a spurious counterexample. A completely lazy abstraction corresponds to using no clusters. Thus, the initial abstraction is simply true. Motivated by this idea, we use a very inexpensive syntactic partitioning to compute a very coarse initial abstraction. This is done to compute initial abstractions of large circuits quickly.

There are many ways to perform a partitioning for an inexpensive abstraction. One simple technique is to create $k$ clusters, each containing exactly one current-state predicate $\pi_{i}$. We follow a variant of this technique: all current-state predicates that contain the 
exact same set of variables are kept in the same partition. This is useful if the given set of predicates contains many mutually exclusive (or related) predicates such as $x=1, x=2, x=3$. Keeping these predicates in separate clusters will result in an exponential number of contradicting abstract states, such as an abstract state in which both $x=1$ and $x=2$ are true. The next-state predicates are not used for computing the abstraction.

Example: Let the set of current-state predicates be $\{x<200, x=$ $100, y=100, z>100\}$. The clusters produced for lazy abstraction are $C_{1}:=\{x<200, x=100\}, C_{2}:=\{y=100\}, C_{3}:=\{z>100\}$.

Once the abstraction of the concrete system is obtained, we modelcheck it using the NuSMV model-checker [18]. If the abstract model satisfies the property, the property also holds on the original, concrete circuit. If the model checking of the abstraction returns false, we obtain a counterexample from the model-checker. In order to check if an abstract counterexample corresponds to a concrete counterexample, a simulation step is performed. If the counterexample cannot be simulated on the concrete model, it is called a spurious counterexample. The elimination of spurious counterexamples from the abstract model is described in the next section.

\section{ABSTRACTION REFINEMENT}

When refining the abstract model, we distinguish between two cases of spurious behavior, as done in [11]: Spurious transitions are abstract transitions which do not have any corresponding concrete transitions. By definition, spurious transitions cannot appear in the most precise abstraction as computed by the eager approach. However, as we noted earlier, computing the most precise abstract model is expensive and thus, we make use of the various partitioning techniques. These techniques can typically result in many spurious transitions. Spurious prefixes are prefixes of the abstract counterexample that do not have a corresponding concrete path. This happens when the set of predicates is not rich enough to capture the relevant behaviors of the concrete system, even for the most precise abstraction.

An abstract counterexample is a sequence of abstract states $\bar{s}(1)$, $\ldots, \bar{s}(l)$, where each abstract state $\bar{s}(j)$ corresponds to a valuation of the $k$ predicates $\pi_{1}, \ldots, \pi_{k}$. The value of $\pi_{i}$ in a state $\bar{s}$ is denoted by $\bar{s}_{i}$. Recall that $\pi_{i}^{\prime}$ denotes the next state version of $\pi_{i}$. In order to check if an abstract transition $\bar{s}$ to $\bar{t}$ can be simulated on the concrete model, we create a SAT instance given by the following equation:

$$
\bigwedge_{i=1}^{k} \pi_{i}=\bar{s}_{i} \wedge R\left(\bar{r}, \bar{r}^{\prime}\right) \wedge \bigwedge_{i=1}^{k} \pi_{i}^{\prime}=\bar{t}_{i}
$$

The equation above is transformed into $\mathrm{CNF}$ and passed to a SAT solver. If the SAT solver detects the equation to be satisfiable, the abstract transition can be simulated on the concrete model. Otherwise, the abstract transition is spurious.

Removing spurious transitions. If the abstract transition is spurious, the CNF instance is unsatisfiable. In this case, we make use of the ZChaff SAT solver [17] for finding a subset of clauses in the CNF instance which is also unsatisfiable(called an unsatisfiable core). It is computed by making use of the proof of unsatisfiability of the SAT instance [22]. We use the unsatisfiable core to determine a subset of existing predicates $\left(\pi_{i}\right)$ which are sufficient to show that the abstract transition is spurious. The spurious transition is removed from the abstract model by adding a constraint in terms of the predicates appearing in the unsatisfiable core.

Example: Consider the abstract transition from $\bar{s}=\left\{b_{1}=0, b_{2}=\right.$ $1\}$ to $\bar{t}=\left\{b_{1}^{\prime}=0, b_{2}^{\prime}=0\right\}$, where $b_{1}, b_{2}$ represent the current state values and $b_{1}^{\prime}, b_{2}^{\prime}$ represent the next state values of predicates $x>2$, $y=3$, respectively. Let the next state functions be $x^{\prime}=y, y^{\prime}=x$.
Observe that in $\bar{s}$, the predicate $y=3$ is true. This implies that $x^{\prime}=3$, and thus, $b_{1}^{\prime}$ must hold in $\bar{t}$. However, $b_{1}^{\prime}$ is false in $\bar{t}$ and thus, the transition from $\bar{s}$ to $\bar{t}$ is spurious. This transition can be eliminated by adding the constraint $\neg\left(\neg b_{1} \wedge b_{2} \wedge \neg b_{1}^{\prime} \wedge \neg b_{2}^{\prime}\right)$ to the abstract model. However, this constraint removes just one spurious transition. By making use of an unsatisfiable core, we can make the constraint more general, thereby eliminating many spurious transitions at the same time. In this example, the cause of the spurious behavior is due to $b_{2}=1$, and $b_{1}^{\prime}=0$. The unsatisfiable core allows us to discover this fact. Now we can eliminate this abstract transition and many more spurious transitions by adding the following constraint to the abstract model: $\neg\left(b_{2} \wedge \neg b_{1}^{\prime}\right)$.

Removing spurious prefixes. In [11], the elimination of spurious prefixes is done by adding a monolithic bit-level predicate. In contrast to that, we make use of weakest preconditions as done in software verification. We generate new word-level predicates from the weakest pre-condition of the given property with respect to the transition function given by the RTL level circuit.

Weakest pre-conditions for Verilog. In software verification, the weakest pre-condition $w p(s t, \gamma)$ of a formula $\gamma$ is usually defined with respect to a statement st (e.g., an assignment). It is the weakest formula whose truth before the execution of $s t$ entails the truth of $\gamma$ after st terminates. In case of hardware, each state transition can be viewed as a statement where the registers are assigned values according to their next-state functions.

Recall that the set of registers that have a next-state function is denoted by $Q$. For example, external inputs do not appear in this set. The next-state function for register $r_{i} \in Q$ is given by $f_{i}(\bar{r})$. We use $\bar{f}$ to denote the vector of the next state functions for the registers in $Q$. For any expression $e$, the expression $e[\bar{x} / \bar{y}]$ denotes the simultaneous substitution of each $x_{i}$ in $e$ by $y_{i}$ from $\bar{y}$. Note that $x_{i}$ and $y_{i}$ might themselves be expressions.

The weakest precondition of the property $\gamma(\bar{r})$ with respect to one concrete transition is defined as follows:

$$
w p_{1}(\bar{f}, \gamma(\bar{r})):=\gamma(\bar{r})[\bar{r} / \bar{f}]
$$

The weakest precondition with respect to $i$ consecutive concrete transitions is defined inductively as follows:

$$
\left.w p_{i}(\bar{f}, \gamma)\right):=w p_{1}\left(\bar{f}, w p_{i-1}(\bar{f}, \gamma)\right)(i>1)
$$

In order to refine a spurious prefix of length $l>0$, we compute $w p_{l}(\bar{f}, \tau)$, where $\tau$ is the safety property we are interested in checking. Intuitively, $\tau$ holds holds after $l$ transitions iff $w p_{l}(\bar{f}, \tau)$ holds before $l$ transitions. Refinement corresponds to adding the boolean expressions occurring in $w p_{l}(\bar{f}, \tau)$ to the existing set of predicates. The abstraction, created with respect to the new set of predicates, results in a model that does not contain this spurious prefix.

Example: Let the property be $x<3$, and the next state function for the register $x$ be $((x<5) ?(x+2): x)$. Suppose we obtain an spurious prefix of length equal to 1 . The weakest pre-condition $w p_{1}$ is given as $(((x<5) ?(x+2): x)<3)$.

Simplifying the weakest pre-conditions. The problem with the approach above is that the predicates generated can become very complex when the spurious prefix is large. This will adversely affect the future iterations of the abstraction refinement loop. In software verification, this problem is solved by computing the weakest pre-condition with respect to the statements appearing in the spurious counterexample trace only. This is not directly applicable to a synchronous circuit.

Instead, we apply a syntactic simplification to the weakest preconditions at each step. The simplification uses data from the abstract error trace. We exploit the fact that many of the control flow 
guards in the Verilog file are also present in the current set of predicates. The abstract trace assigns truth values to these predicates in each abstract state. In order to simplify the weakest pre-conditions, we substitute the guards in the weakest pre-conditions with their truth values. Furthermore, we only add the atomic predicates in the weakest pre-condition as the new predicates.

Example: Suppose the guard $x<5$ is present in the current set of predicates. Let the value of $x<5$ in an abstract state $\bar{s}$ be true. The weakest pre-condition given as $(((x<5) ?(x+2): x)<3)$, can be simplified in $\bar{s}$, by substituting the value of $x<5$. This results in a new predicate $x+2<3$ (or $x<1$ ).

With weakest pre-condition simplification, it is not always enough to compute the weakest pre-condition of the given property for refinement. One needs to identify a subset of existing predicates, whose weakest pre-condition must be computed for removing the spurious behavior. This is done by simulating the spurious prefix and picking the predicates that appear in the unsatisfiable core. If a copy of predicate $p$ in cycle $k$ appears in the unsatisfiable core, then we compute the weakest pre-condition of $p$ for $k$ steps.

\section{EXPERIMENTAL RESULTS}

The experiments are performed on a $1.5 \mathrm{GHZ}$ AMD machine with $3 \mathrm{~GB}$ of memory running Linux. A time limit of one hour and a memory limit of $700 \mathrm{MB}$ was set for each run. We compare our technique against a non-commercial version of the Cadence SMV model checker [12]. The Cadence SMV tool is a net-list based model checker; it reads in a RTL-Level circuit and generates a nextstate function for each latch in the circuit. In contrast to that, we generate a next state function for each register.

\subsection{Benchmarks and Properties verified}

Our benchmarks are taken from the Instruction Cache Unit (ICU), and the Instruction Cache RAM (ICRAM) unit of the Sun PicoJava II microprocessor [20]. The ICU fetches the instructions from the instruction cache and passes them to the decode unit. We checked the property that in case of a cache read miss the ICU controller implementation simulates a miss state transition diagram given in the picoJava-II micro-architecture guide [20].

The ICRAM maintains a RAM of size 16KB (organized as 2048 entries of 64 bits) each. If the write is enabled (icu_ram_we[1:0] = 2 'b10), then the value of data input (icu_din) is written to the higher 32 bits of the location addressed by the input address (icu_addr). This functionality of the ICRAM was encoded in form of a safety property using the current and the next state of the variables. Observe that the property depends on the contents of the RAM. Thus, even after applying the techniques such as localization reduction, the system will have $16 \mathrm{~KB}(16 \times 1024 \times 8)$ latches. In order to simplify the problem, we verified the property for the RAM of sizes 512 byte, $1 \mathrm{~KB}, 2 \mathrm{~KB}$, and $4 \mathrm{~KB}$. These benchmarks are denoted as M512B, M1KB, M2KB, M4KB, in the Table 1, respectively.

The benchmarks starting with "AR" perform arithmetic operations on two registers $a$ and $b$ in each clock cycle. The next state functions of $a$ and $b$ are given as follows: $a^{\prime}:=(a<100) ?(a+b): a$ and $b^{\prime}:=a$. Initial values of these registers are 1 and 0 , respectively. We check the property that $b \leq a$ in each clock cycle. The benchmarks AR100, AR200, AR500, AR1000 in Table 1 are variants of this circuit obtained by increasing the size of the registers $a$ and $b$.

The experimental results are summarized in Table 1. The "Latches" column contains the total number of latches in the cone of influence of the property. We compare two different techniques for verifying these benchmarks. The columns marked with "Predicate Abstraction" contain the results of applying the predicate abstraction and refinement techniques discussed in this paper. The "Time", "Abs",
"MC", and "Ref" columns contain the total time, followed by the breakup of the total time into the time taken by abstraction, model checking, and refinement including simulation. The "P/I" column contains the final number of predicates followed by the total number of iterations.

The results of running Cadence SMV are given in the "CSMV" column. Of the various options to Cadence SMV, we found the counterexample-based abstraction refinement option -absref 3 [6] to result in the best performance when checking the picoJava-II properties. We report the total time taken by Cadence SMV when running with this option.

\begin{tabular}{|l|c|cccc|c|c|}
\hline Bench- & Latches & \multicolumn{5}{|c|}{ Predicate Abstraction } & CSMV \\
\cline { 3 - 6 } mark & & Time & Abs & MC & Ref & P/I & Time \\
\hline ICU & 28 & 1.3 & 0.6 & 0.1 & 0.6 & $5 / 1$ & 0.1 \\
M512B & 4137 & 107.1 & 2.2 & 0.8 & 104.1 & $3 / 8$ & 2.3 \\
M1KB & 8234 & 180.8 & 9.3 & 0.8 & 170.7 & $3 / 8$ & 7.5 \\
M2KB & 16427 & 450.7 & 24 & 0.9 & 425.3 & $3 / 8$ & 25.0 \\
M4KB & 32796 & 843.3 & 37 & 0.8 & 805.5 & $3 / 8$ & - \\
AR100 & 202 & 3.5 & 2.8 & 0.12 & 0.55 & $3 / 3$ & 182.4 \\
AR2 0 0 & 402 & 9.6 & 8.4 & 0.12 & 1.1 & $3 / 3$ & 2147 \\
AR50 0 & 1002 & 32.2 & 29.3 & 0.12 & 2.8 & $3 / 3$ & $*$ \\
AR1000 & 2002 & 122.6 & 116.8 & 0.16 & 5.6 & $3 / 3$ & $*$ \\
\hline
\end{tabular}

Table 1: Experimental results: All runtimes are in seconds. A "*» indicates a timeout of 1 hour. A "." indicates the model checker terminated due to the large number of BDD variables.

\subsection{Summary of Results}

On the ICU benchmark, Cadence SMV outperforms predicate abstraction. Since the state space of this benchmark is very small, no abstraction is necessary. On the M512B, M1KB, and M2KB benchmarks, the runtime of Cadence SMV is better than the predicate abstraction runtime. However, Cadence SMV is not able to handle the M4KB benchmark which has a much larger state space. Cadence SMV timeouts on the AR500 and the AR1000 benchmarks, while the predicate abstraction method is able to complete these benchmarks with better runtimes. Some of the inferences drawn from these observations are as follows:

- The runtime of Cadence SMV grows exponentially with each newly added latch. This trend is visible in the AR100 to AR1000 benchmarks. In these benchmarks, Cadence SMV is not able to reduce the number of latches in the abstract model created, making the model checking step expensive.

- Using predicate abstraction the size of the abstract model remains constant even when the number of latches are increased. This is because for many properties the number of word-level predicates needed for proof does not grow, as the sizes of the registers appearing in the property is increased. This trend is visible in the $\mathrm{M}^{*}$ and the $\mathrm{AR}^{*}$ benchmarks, where the number of predicates needed to prove the property does not change as the number of latches is increased. Thus, the model checking (MC) time is similar across all $\mathrm{M}^{*}$ benchmarks and across the $\mathrm{AR}^{*}$ benchmarks.

- The computation of the abstract model using predicate abstraction requires the use of a decision procedure, which is a SAT solver in our case. In general, the problem of computing the precise existential abstraction (Eqn. 1) is itself exponential in the number of predicates and the size of the transition relation (number of latches). However, this complexity is not observed in our experiments due to two reasons: 1) the use of state of art SAT solvers like ZChaff [17] and Siege [19], 2) the use of our predicate partitioning technique (Sec. 4 ) to handle the large number of predicates. The experimental results indicate that the 

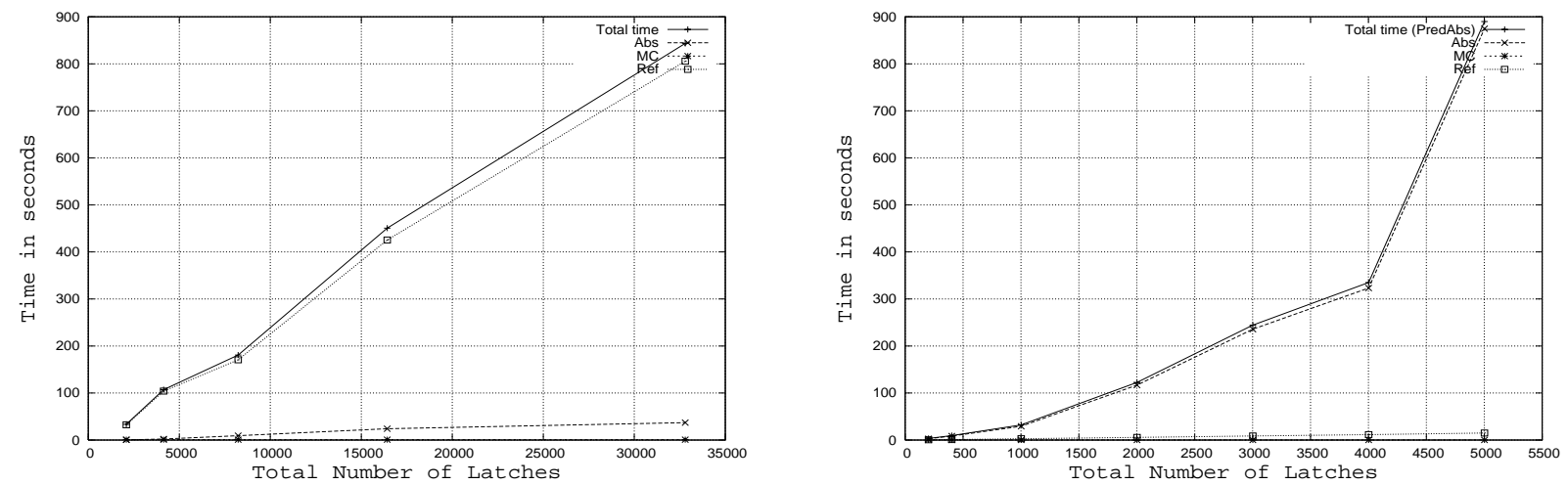

Figure 2: Runtime of the predicate abstraction and refinement with respect to number of latches: (a) $M^{*}$ benchmarks (b) $A R^{*}$ benchmarks

abstraction computation time does not grow exponentially with each newly added latch.

A plot of the total time needed by the predicate abstraction technique compared to the number of latches is given in Fig. 2(a) and Fig. 2(b) for the $\mathrm{M}^{*}$ and the $\mathrm{AR}^{*}$ benchmarks, respectively. Observe that the runtime does not increase exponentially with number of latches. These experiments support the hypothesis that the iterative predicate abstraction and refinement can scale to circuits involving thousands of latches.

Predicate partitioning techniques. We experimented with two different techniques for creating predicate clusters (section 4), namely cone partitioning and partitioning for lazy abstraction. Both techniques are complementary to each other. Cone partitioning attempts to keep all related predicates together, thus, the abstract model produced is more precise as compared to lazy abstraction. However, the time taken for abstraction using cone partitioning can become a bottleneck. In such cases, lazy abstraction works well if the property can be checked using a coarse abstract model. Cone partitioning is used for $\mathrm{AR}^{*}$ benchmarks, while the lazy abstraction is used for $\mathrm{M}^{*}$ benchmarks. Observe that the total time is dominated by the abstraction time in case of $\mathrm{AR}^{*}$ benchmarks, and the refinement time in case of the $\mathrm{M}^{*}$ benchmarks. Additional experiments can be found in our technical report [9].

Performance on Vapor benchmarks. The Vapor tool [1] performs abstraction of the Verilog models to the CLU language [4] for verification. In [1], Vapor was used to verify control related properties of the ITC 99 circuits. We found that all the 21 properties of the ITC-b13 benchmark are proved trivially using predicate abstraction. Of the 21 properties, 18 properties can be proved using two or less predicates. The time taken is less than one second and no refinement iterations are needed. The remaining three properties are proved in less than 20 seconds, using 8 or fewer predicates, with four refinement iterations. The other ITC 99 circuits reported in [1] are also handled in a straightforward way.

\section{CONCLUSIONS}

Localization reduction fails if the property depends on too many latches. This requires a stronger abstraction technique. This paper presents novel algorithms for computing and refining predicate abstractions of circuits given in RTL Verilog using SAT.

There are two challenges when using predicate abstraction on Verilog: 1) the computation of the abstract model, and 2) how to obtain good predicates. We address the first challenge by introducing predicate partitioning, a hybrid between lazy abstraction [15] and eager abstraction. We make use of unsatisfiable cores of SAT instances in order to eliminate multiple spurious transitions caused by the imprecise abstraction.
In order to obtain the right set of predicates, we compute new word-level predicates by using weakest pre-conditions of Verilog RTL. Weakest pre-conditions are commonly used in the software domain. However, this technique was not applied to hardware before, despite of the fact that high-level RTL closely resembles languages like ANSI-C. Our experimental results show that this technique is very effective in discovering new word-level predicates for refinement. On the large benchmarks, our new algorithm scales well with the design size and clearly outperforms existing algorithms that use localization reduction.

\section{REFERENCES}

[1] Z. S. Andraus and K. A. Sakallah. Automatic abstraction and verification of Verilog models. In DAC, pages 218-223, 2004.

[2] T. Ball, B. Cook, S. Das, and S.K. Rajamani. Refining approximations in software predicate abstraction. In TACAS, pages 388-403, 2004

[3] T. Ball and S.K. Rajamani. Boolean programs: A model and process for software analysis. Technical Report 2000-14, Microsoft Research, 2000

[4] R. Bryant, S. Lahiri, and S. Seshia. Modeling and verifying systems using a logic of counter arithmetic with lambda expressions and uninterpreted functions. In $C A V, 2002$.

[5] P. Chauhan, E.M. Clarke, J. Kukula, S. Sapra, H. Veith, and D. Wang. Automated abstraction refinement for model checking large state spaces using sat based conflict analysis. In FMCAD, 2002.

[6] E. Clarke, O. Grumberg, S. Jha, Y. Lu, and Veith H. Counterexample-guided abstraction refinement. In $C A V$, pages $154-169,2000$.

[7] E. Clarke, O. Grumberg, and D.E. Long. Model checking and abstraction. In POPL, 1992.

[8] E. Clarke, O. Grumberg, and D. Peled. Model Checking. MIT Press, 1999.

[9] E. Clarke, H. Jain, and D. Kroening. Predicate Abstraction and Refinement Techniques for Verifying Verilog. Technical Report CMU-CS-04-139, Carnegie Mellon University, 2004.

[10] E. Clarke, D. Kroening, N. Sharygina, and K. Yorav. Predicate abstraction of ANSI-C programs using SAT. Formal Methods In System Design, 25, 2004.

[11] E. Clarke, M. Talupur, and D. Wang. SAT based predicate abstraction for hardware verification. In SAT, 2003.

[12] www-cad.eecs.berkeley.edu/ kenmcmil/smv/.

[13] S. Das and D. Dill. Successive approximation of abstract transition relations. In LICS, 2001. June 2001, Boston, USA.

[14] S. Graf and H. Saidi. Construction of abstract state graphs with PVS. In $C A V$, volume 1254, pages 72-83, 1997.

[15] T. A. Henzinger, R. Jhala, R. Majumdar, and G. Sutre. Lazy abstraction. In $P O P L$, pages 58-70, 2002.

[16] R.P. Kurshan. Computer-aided verification of coordinating processes: the automata-theoretic approach. Princeton University Press, 1994.

[17] M. Moskewicz, C. Madigan, Y. Zhao, L. Zhang, and S. Malik. Chaff: Engineering an efficient SAT solver. In DAC, pages 530-535, 2001.

[18] http://nusmv.irst.itc.it/.

[19] http://www.cs.sfu.ca/ loryan/personal.

[20] http://www.sun.com/processors/technologies.html.

[21] D. Wang, P. Ho, J. Long, J. Kukula, Y. Zhu, T. Ma, and R. Damiano. Formal property verification by abstraction refinement with formal, simulation and hybrid engines. In $D A C$, pages 35-40, 2001.

[22] L. Zhang and S. Malik. Extracting small unsatisfiable cores from unsatisfiable boolean formulas. In SAT, 2003. 